\title{
THE MULTIPLICATIVE COUSIN PROBLEM AND A ZERO SET FOR THE NEVANLINNA CLASS IN THE POLYDISC
}

\author{
BY \\ SERGIO E. ZARANTONELLO
}

\begin{abstract}
Let $\Omega$ be a polydomain in $\mathbf{c}^{n}$, the Nevanlinna class $N(\Omega)$ consists of all holomorphic functions $f$ in $\Omega$ such that $\log ^{+}|f|$
\end{abstract} has an $n$-harmonic majorant in $\Omega$. Let $U^{n}$ be the open unit polydisc $\left\{z \in \mathrm{C}^{n}:\left|z_{1}\right|<1, \cdots,\left|z_{n}\right|<1\right\}$. THEOREM 1. Given an open covering $\left(\Omega_{\alpha}\right)_{\alpha \in A}$ of the closure $\bar{U}^{n}$ of the polydisc, consisting of polydomains, and for each $\alpha \in A$ a function $f_{\alpha} \in N\left(\Omega_{\alpha} \cap U^{n}\right)$ such that for all $\alpha, \beta \in A, f_{\alpha} f_{\beta}^{-1}$ is an invertible element of $N\left(\Omega_{\alpha} \cap \Omega_{\beta} \cap U^{n}\right)$. There exists a function $F \in N\left(U^{n}\right)$ such that for all $\alpha \in A, F f_{\alpha}^{-1}$ is an invertible ele. ment of $N\left(\Omega_{\alpha} \cap U^{n}\right)$. This result enables us to find the following sufficient condition for the zero sets of $N\left(U^{n}\right)$ : THEOREM 2. Let $f$ be a holomorphic function in $U^{n}, n>2$. If there exists a constant $0<r<1$ and a continuous function $\eta:[r, 1) \rightarrow[r, 1)$ such that

$$
\left|z_{n}\right|<\eta\left(\frac{\left|z_{1}\right|+\cdots+\left|z_{n-1}\right|}{n-1}\right)
$$

for all points $\left(z_{1}, \cdots, z_{n}\right)$ satisfying $\left|z_{1}\right|>r, \cdots,\left|z_{n}\right|>r$ and $f\left(z_{1}, \cdots, z_{n}\right)=0$, then $f$ has the same zeros as some function $F \in N\left(U^{n}\right)$. In the above if $\overline{\lim }_{\lambda \rightarrow 1} \eta(x)<1$, then $Z(f)$ is a Rudin variety in which case there is a bounded holomorphic function with the same zeros as $f$.

I. Introduction. The theory of functions in the polydisc $U^{n}$ has many peculiarities of its own and is not a mere generalization of the one variable case. This becomes evident when we consider the zero set problem. In complex dimension one, the Blaschke condition is necessary and sufficient for a sequence to be the zero set of a bounded holomorphic function in the disc; also the zero sets of $H^{\infty}(U)$ and of $N(U)$ are the same. In higher complex dimensions

Received by the editors January 23, 1973 and, in revised form, October 16, 1973.

AMS (MOS) subject classifications (1970). Primary 32A30; Secondary 31C10, 31A05.

Key words and phrases. Nevanlinna class, polydisc, multiplicative Cousin problem, holomorphic, $n$-harmonic, Poisson kernel, Poisson integral, Green's function. 
this is no longer true. The zero sets of $H^{\infty}\left(U^{n}\right)$ and of $N\left(U^{n}\right)$ (the Nevanlinna class of functions in the polydisc) are different (Rudin [4]), and the generalized Blaschke condition is necessary but no longer sufficient for an analytic set (the zero set of a holomorphic function) to be the zero set of a function in $N\left(U^{n}\right)$ (Chee [2] , [3]).

In this article we find a geometric condition on an analytic set so that it is the zero set of a function in $N\left(U^{n}\right)$. Our method of attack is first to solve the multiplicative Cousin problem for $N\left(U^{n}\right)$ and then to find the restriction on the geometry of the analytic set.

The motivation for this approach is the connection between the possibility of solving the multiplicative Cousin problem with bounded data in $U^{n}$ (Stout [6]), and the sufficient condition for the zero sets of $H^{\infty}\left(U^{n}\right)$ given by Rudin in [5]. To establish this connection, first observe that if an analytic set in $U^{n}$ is "locally" determined by bounded holomorphic functions, the quotient of any two of which is an invertible bounded holomorphic function in the intersection of their domains, then the solution to the multiplicative Cousin problem is a bounded holomorphic function whose zero set is the given analytic set. In complex dimension two it is easy to see [7] that the condition given by Rudin on an analytic set implies that it is locally the zero set of bounded holomorphic functions with the compatibility conditions mentioned above. Although combined this gives a lengthier proof, the method of first solving a Cousin type problem, and then obtaining a condition on the zero set can be used in other contexts. In [7] it was used to extend Rudin's result to the product of two annuli. In the present article our concern is with the Nevanlinna class $N\left(U^{n}\right)$.

This paper consists of three sections. The multiplicative Cousin problem for $N\left(U^{n}\right)$ is solved in $\S I I I$, from this it follows that if $f$ is holomorphic in $U^{n}$, and its zero set is locally determined by bounded holomorphic functions (there is an open covering $\left\{V_{\alpha}\right\}$ of the closure of $f^{-1}(0)$, and for each $\alpha$, a bounded holomorphic function $f_{\alpha}$ with the same zeros as $f$ in $V_{\alpha} \cap U^{n}$ ), then $f$ has the same zeros as some function $F$ in $N\left(U^{n}\right)$. Observe that we do not require that $f_{\alpha} f_{\beta}^{-1}$ be an invertible element of $H^{\infty}\left(V_{\alpha} \cap V_{\beta} \cap U^{n}\right)$; if we did then $F$ would be in $H^{\infty}\left(U^{n}\right)$.

In $\S I V$ we obtain a geometric condition on the zero set of a holomorphic function $f$ in $U^{n}$ so that it is locally given by bounded holomorphic functions, and thus agrees with the zero set of some function $F$ in $N\left(U^{n}\right)$. Actually, not only will the zero sets agree, but also $F$ will recapture the zeros with the same multiplicity regarding $f$. Finally in §II we prove a technical result which we use in §III: That in an appropriate polydomain, if a positive $n$-subharmonic function has "local" $n$-harmonic majorant then it must have a global one.

This work was based on the author's doctoral thesis under the direction of 
Professor Walter Rudin, whose guidance and encouragement he wishes to acknowledge.

\section{Subharmonic and $n$-subharmonic functions.}

(2.1) Preliminaries. In what follows, by an analytic arc we will mean a simple regular analytic arc; similarly a closed analytic curve will be a simple regular analytic closed curve.

A domain will be a connected open set $\Omega \subset \mathrm{C}$, its topological boundary will be denoted by $\partial \Omega$, and its closure by $\bar{\Omega}$. If $\Omega$ is a bounded domain whose boundary consists of finitely many separated simple closed curves, each one composed of a finite number of analytic arcs, it has a Green's function $g^{\Omega}\left(z, z_{0}\right)$, and the following conditions hold:

(i) If $\Gamma$ is an open analytic arc in $\partial \Omega$, then $\left|\nabla g^{\Omega}\left(z, z_{0}\right)\right|$ is a strictly positive and continuous function of $z$ in $\Gamma$.

(ii) If $\phi$ is subharmonic in a neighborhood of $\bar{\Omega}$, its least harmonic majorant in $\Omega$ will be $u_{\phi}^{\Omega}(z)=\int_{\partial \Omega} \phi(\omega)\left|\nabla g^{\Omega}(z, \omega)\right| d m(\omega)$.

In (i) and (ii) $\nabla g^{\Omega}\left(z, z_{0}\right)$ denotes the gradient of $g^{\Omega}\left(z, z_{0}\right)$. Its absolute value $\left|\nabla g^{\Omega}\left(z, z_{0}\right)\right|$ is equal to the interior normal derivative of $g^{\Omega}\left(z, z_{0}\right)$. The measure $m$ in (ii) is the measure induced by $1 / 2 \pi$ arc length on $\partial \Omega$.

If a subharmonic function $\phi$ has a harmonic majorant in a domain $\Omega$, its least harmonic majorant (whether it is given as in (ii) or not) will be denoted by $u_{\phi}^{\Omega}$. Suppose that $\Omega_{n}$ is an ascending sequence of subdomains of $\Omega$, whose union is $\Omega$, and $\phi$ is subharmonic in $\Omega$ with least harmonic majorants $u_{\phi} \Omega_{n}$ in each $\Omega_{n}$. Then $u_{\phi}^{\Omega_{n}}$ is an increasing sequence of harmonic functions which either converges to $u_{\phi}^{\Omega}$, if $u_{\phi}^{\Omega}$ exists, or diverges at every point.

We will call the boundary of a domain nice, if it consists of finitely many separated simple closed curves, each composed of a finite number of analytic arcs.

(2.2) LEMMA. Let $\Omega$ be a bounded domain, bounded by finitely many separated analytic closed curves. Let $\Omega^{1}$ and $\Omega^{2}$ be subdomains of $\Omega$ with nice boundaries, such that:

(i) $\Omega=\Omega^{1} \cup \Omega^{2}$,

(ii) $\partial \Omega=\operatorname{int}\left(\partial \Omega^{1} \cap \partial \Omega\right) \cup \operatorname{int}\left(\partial \Omega^{2} \cap \partial \Omega\right)$, where int indicates interior with respect to $\partial \Omega$.

If $z_{0} \in \Omega^{1} \cap \Omega^{2}$, there exists a constant $A>0$, such that for any positive subharmonic function $\phi$ in a neighborhood of $\bar{\Omega}$ :

$$
u_{\phi}^{\Omega}\left(z_{0}\right) \leqslant A\left[u_{\phi}^{\Omega^{1}}\left(z_{0}\right)+u_{\phi}^{\Omega^{2}}\left(z_{0}\right)\right]
$$


If $\partial^{\prime} \subset^{\text {compact }} \operatorname{int}\left(\partial \Omega^{1} \cap \partial \Omega\right), \partial^{\prime \prime} \subset^{\text {compact }} \operatorname{int}\left(\partial \Omega^{2} \cap \partial \Omega\right)$ are such that $\partial^{\prime} \cup \partial^{\prime \prime}=\partial \Omega$, then a value for the constant $A$ is

$$
A=\frac{\sup _{\omega \in \partial \Omega}\left|\nabla g^{\Omega}\left(z_{0}, \omega\right)\right|}{\min \left(\inf _{\omega \in \partial^{\prime}}\left|\nabla g^{\Omega^{1}}\left(z_{0}, \omega\right)\right|, \inf _{\omega \in \partial^{\prime \prime}}\left|\nabla g^{\Omega^{2}}\left(z_{0}, \omega\right)\right|\right)}
$$

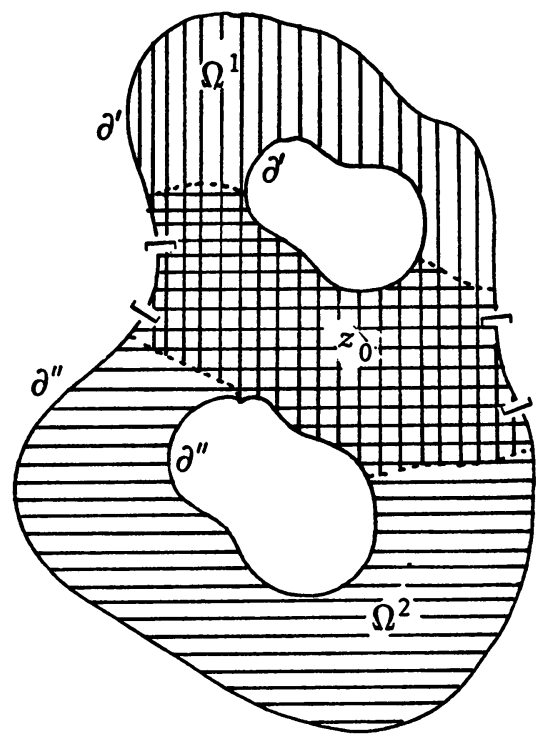

ProOF. Let $\partial^{\prime} \subset^{\text {compact }} \operatorname{int}\left(\partial \Omega^{1} \cap \partial \Omega\right)$ and $\partial^{\prime \prime} \subset^{\text {compact }} \operatorname{int}\left(\partial \Omega^{2} \cap \partial \Omega\right)$ such that $\partial^{\prime} \cup \partial^{\prime \prime}=\partial \Omega$. Fix $z_{0} \in \Omega^{1} \cap \Omega^{2}$.

Let

$$
\begin{gathered}
M=\sup _{\omega \in \partial \Omega}\left|\nabla g^{\Omega}\left(z_{0}, \omega\right)\right|, \quad m^{\prime}=\inf _{\omega \in \partial^{\prime}} \nabla g^{\Omega^{1}}\left(z_{0}, \omega\right) \mid, \\
m^{\prime \prime}=\inf _{\omega \in \partial^{\prime \prime}} \nabla g^{\Omega^{2}}\left(z_{0}, \omega\right) \mid, \quad m=\min \left(m^{\prime}, m^{\prime \prime}\right) .
\end{gathered}
$$

Since $\partial \Omega$ is analytic, and both $\operatorname{int}\left(\partial \Omega \cap \partial \Omega^{1}\right)$ and $\operatorname{int}\left(\partial \Omega \cap \partial \Omega^{2}\right)$ are unions of open analytic arcs, it follows that $0<m<\infty$ and $0<M<\infty$. Hence for any positive subharmonic $\phi$ in a neighborhood of $\bar{\Omega}$ we have 


$$
\begin{aligned}
u_{\phi}^{\Omega}\left(z_{0}\right) & =\int_{\partial \Omega=\partial^{\prime} \cup \partial^{\prime \prime}} \phi(\omega)\left|\nabla g^{\Omega}\left(z_{0}, \omega\right)\right| d m(\omega) \\
& \leqslant \int_{\partial} \phi(\omega)\left|\nabla g^{\Omega}\left(z_{0}, \omega\right)\right| d m(\omega)+\int_{\partial^{\prime \prime}} \phi(\omega)\left|\nabla g^{\Omega}\left(z_{0}, \omega\right)\right| d m(\omega) \\
& \leqslant M \int_{\partial^{\prime}} \phi(\omega) d m(\omega)+M \int_{\partial^{\prime \prime}} \phi(\omega) d m(\omega) \\
\leqslant & \frac{M}{m}\left[\int_{\partial^{\prime}} \phi(\omega)\left|\nabla g^{\Omega^{1}}\left(z_{0}, \omega\right)\right| d m(\omega)+\int_{\partial "} \phi(\omega)\left|\nabla g^{\Omega^{2}}\left(z_{0}, \omega\right)\right| d m(\omega)\right] \\
\leqslant & \frac{M}{m}\left[\int_{\partial \Omega^{1}} \phi(\omega)\left|\nabla g^{\Omega^{1}}\left(z_{0}, \omega\right)\right| d m(\omega)\right. \\
& \left.\quad+\int_{\partial \Omega^{2}} \phi(\omega)\left|\nabla g^{\Omega^{2}}\left(z_{0}, \omega\right)\right| d m(\omega)\right] \\
= & \frac{M}{m}\left[u_{\phi}^{\Omega^{1}}\left(z_{0}\right)+u_{\phi}^{\Omega^{2}}\left(z_{0}\right)\right],
\end{aligned}
$$

which proves our claim.

Our next goal is to extend (2.2) when $\phi$ is positive subharmonic in $\Omega$ (but not necessarily defined in a neighborhood of $\bar{\Omega}$ ). In order to do so, we will need the following lemma.

(2.3) Lemma. Let $U$ be a domain bounded by finitely many separated closed analytic curves, and $V$ be a subdomain of $U$ with a nice boundary. Fix $z_{0} \in V$, and define, for each integer $n>0, U_{n}=\left\{z \in U: g^{U}\left(z, z_{0}\right)>1 / n\right\}$ and $V_{n}=V \cap U_{n}$.

If $\Gamma$ is an open analytic arc common to both $\partial U$ and $\partial V$, and $K$ is a compact subset of $\Gamma$, there exist an integer $n_{0}$ and an open set $W$ containing $K$, such that for each $n \geqslant n_{0}$ the Green's functions $g^{V} n\left(z, z_{0}\right)$ and $g^{V}\left(z, z_{0}\right)$ can be extended to harmonic functions in $W$. Moreover $W$ can be chosen so that on $W$ these extensions are uniformly bounded.

Proof. Denote the disc centered at the origin and of radius $R$ by $D(0, R)$. Let $V^{\prime}$ be a subdomain of $D(0,1)$, and $\Gamma^{\prime}$ an open arc in $\partial D(0,1) \cap \partial V^{\prime}$. Let $r_{n}$ be an increasing sequence approaching 1 , and define $V_{n}^{\prime}=V^{\prime} \cap D\left(0, r_{n}\right)$. If $\widetilde{V}_{n}^{\prime}$ denotes the union of $V_{n}^{\prime}$, its reflection with respect to $\partial D\left(0, r_{n}\right)$ and $V^{\prime} \cap$ $\partial D\left(0, r_{n}\right)$, then any harmonic function in $V_{n}^{\prime}$ which vanishes on $V_{n}^{\prime} \cap \partial D\left(0, r_{n}\right)$ can be extended to a harmonic function in $\widetilde{V}_{n}^{\prime}$ (by the reflection principle). It is clear that if $K^{\prime}$ is a compact subset of $\Gamma^{\prime}$, one can find an open set $W^{\prime}$ containing $K^{\prime}$ and an integer $n_{0}$ such that $W^{\prime} \subset \widetilde{V}_{n}^{\prime}$, for every $n \geqslant n_{0}$. Thus, any harmonic 
function in $V_{n}^{\prime}$ which vanishes on $V^{\prime} \cap \partial D\left(0, r_{n}\right)$ can be extended to a harmonic function in $W^{\prime}$, whenever $n \geqslant n_{0}$.

Suppose that $U, V, \Gamma, K$, etc. are as in the statement of the lemma. The boundary of $U$ is analytic, so we can extend (by reflection) $g^{U}\left(z, z_{0}\right)$ to a harmonic function in a domain containing $\bar{U}-\left\{z_{0}\right\}$. Let $C$ be the component of $\partial U$ containing $\Gamma$, then if $N$ is sufficiently large there will be components $C_{N}$ of $\left\{z: g^{U}\left(z, z_{0}\right)=1 / N\right\}$ and $C_{-N}$ of $\left\{z: g^{U}\left(z, z_{0}\right)=-1 / N\right\}$, both closed analytic curves, enclosing a doubly connected domain $D$ which contains $C$ on which

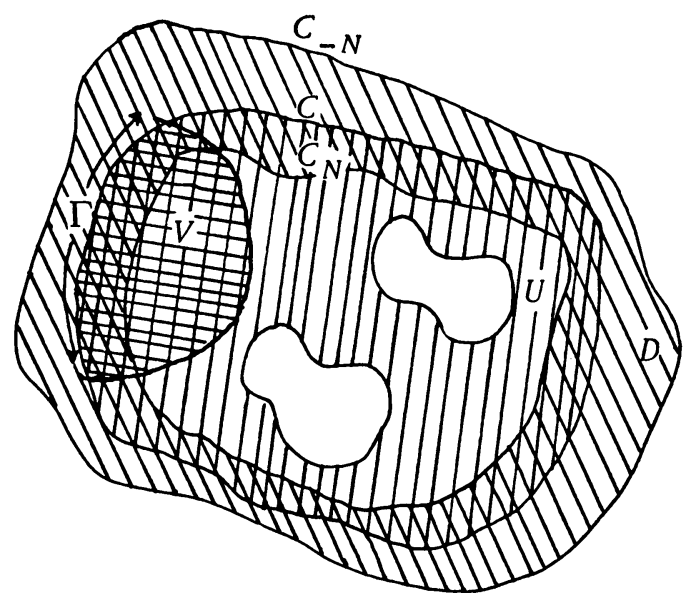

$-1 / N<g^{U}\left(z, z_{0}\right)<+1 / N$. For the sake of simplicity denote $g^{U}\left(z, z_{0}\right)$ by $g(z)$. Since $g$ is harmonic in $D$ with continuous boundary values $1 / N$ on $C_{N}$ and $-1 / N$ on $C_{-N}, 1 / 2 N g(z)+1 / 2$ is the harmonic measure of $C_{-N}$ with respect to $D$. It can be seen (p. 247 of [1]) that if $g^{*}$ is the multivalued harmonic conjugate of $g$ in $D$, there exists a constant $c<0$ such that $\Phi=$ $\exp c\left(g+i g^{*}\right)$ maps $D$, one-to-one conformally, onto the annulus $\left\{\omega \in \mathrm{C}: e^{c / N}<|\omega|<e^{-c / N}\right\}$. Let $V^{\prime}=\Phi(V \cap D), V_{n}^{\prime}=\Phi\left(V_{n} \cap D\right), \Gamma^{\prime}=$ $\Phi(\Gamma)$, and $K^{\prime}=\Phi(K)$. Then $V^{\prime}$ is a subdomain of $D(0,1)$ and $\Gamma^{\prime}$ is an open $\operatorname{arc}$ in $\partial V^{\prime} \cap \partial D(0,1)$, also $V_{n}^{\prime}=V^{\prime} \cap D\left(0, e^{c / n}\right)$, if $n>N$. Since we are in the same setting as in the beginning of the proof, there exist $n_{0}>N$ and an open set $W^{\prime}$ containing $K^{\prime}$, such that any harmonic function in $V_{n}^{\prime}, n \geqslant$ $n_{0}$, vanishing in $\partial D\left(0, e^{c / n}\right)$, can be extended to a harmonic function in $W^{\prime}$. If we let $W=\Phi^{-1}\left(W^{\prime}\right)$, it will follow that each $g^{V_{n}}\left(z, z_{0}\right)$ can be extended to a harmonic function on $W$ whenever $n \geqslant n_{0}$. Also, since each $g^{V_{n}}$ is extended by reflection, and in $D \cap V_{n}$ (empty, unless $n>N$ ) $0<g^{V}<g^{U}<1 / N<$ 1 , we will have for the extension of $g^{V_{n}}$ (which we also denote by $g^{V_{n}}$ ) that $\left|g^{V}\left(z, z_{0}\right)\right|<1 / N<1$ whenever $z \in W$ and $n \geqslant n_{0}$.

We now prove an extension of (2.2). 
(2.4) Lemma. Let $\Omega, \Omega^{1}, \Omega^{2}$ be as in (2.2). Fix $z_{0} \in \Omega^{1} \cap \Omega^{2}$ and define $\Omega_{n}=\left\{z \in \Omega: g^{\Omega}\left(z, z_{0}\right)>1 / n\right\}, \Omega_{n}^{1}=\Omega^{1} \cap \Omega_{n}, \Omega_{n}^{2}=\Omega^{2} \cap \Omega_{n}$.

There exist an integer $n_{0}$ and a constant $A>0$, such that for any positive subharmonic function $\phi$ in $\Omega$ :

$$
u_{\phi}^{\Omega n}\left(z_{0}\right) \leqslant A\left[u_{\phi}^{\Omega_{n}^{1}}\left(z_{0}\right)+u_{\phi}^{\Omega_{n}^{2}}\left(z_{0}\right)\right] .
$$

If both $u_{\phi}^{\Omega^{1}}$ and $u_{\phi}^{\Omega^{2}}$ exist, so does $u_{\phi}^{\Omega}$, and

$$
u_{\phi}^{\Omega}\left(z_{0}\right) \leqslant A\left[u_{\phi}^{\Omega 1}\left(z_{0}\right)+u_{\phi}^{\Omega^{2}}\left(z_{0}\right)\right] .
$$

PROOF. If $n$ is sufficiently large, the boundary of $\Omega_{n}$ will consist of separated analytic closed curves, both $\Omega_{n}^{1}$ and $\Omega_{n}^{2}$ will have nice boundaries, and the union of the interiors (with respect to $\partial \Omega_{n}$ ) of $\partial \Omega_{n} \cap \partial \Omega_{n}^{1}$ and of $\partial \Omega_{n} \cap \partial \Omega_{n}^{2}$ will be all of $\partial \Omega_{n}$. So by (2.2), if $\phi$ is any positive subharmonic function in $\Omega$ :

$$
u_{\phi}^{\Omega} n\left(z_{0}\right) \leqslant A_{n}\left[u_{\phi}^{\Omega 1}{ }^{1}\left(z_{0}\right)+u_{\phi}^{\Omega^{2}} n_{\left(z_{0}\right)}\right)
$$

Let $\partial_{n}^{\prime} \subset^{\text {compact }} \operatorname{int}\left(\partial \Omega_{n} \cap \partial \Omega_{n}^{1}\right), \partial_{n}^{\prime \prime} \subset^{\text {compact }} \operatorname{int}\left(\partial \Omega_{n} \cap \partial \Omega_{n}^{2}\right)$, and $\partial_{n}^{\prime} \cup \partial_{n}^{\prime \prime}=\partial \Omega_{n}$, then the constant $A_{n}$ in (2.4.1) will be given by $A_{n}=$ $M_{n} / m_{n}$, where $M_{n}=\sup _{z \in \partial \Omega_{n}}\left|\nabla g^{\Omega_{n}}\left(z, z_{0}\right)\right|, m_{n}^{\prime}=\inf _{z \in \partial_{n}^{\prime}}\left|\nabla g^{\Omega_{n}}\left(z, z_{0}\right)\right|$, $m_{n}^{\prime \prime}=\inf _{z \in \partial_{n}^{\prime \prime}}\left|\nabla g^{\Omega_{n}^{2}}\left(z, z_{0}\right)\right|$, and $m_{n}=\min \left(m_{n}^{\prime}, m_{n}^{\prime \prime}\right)$.

Choose now $\partial^{\prime} \subset^{\text {compact }} \operatorname{int}\left(\partial \Omega \cap \partial \Omega^{1}\right)$ and $\partial^{\prime \prime} \subset^{\text {compact }} \operatorname{int}\left(\partial \Omega \cap \partial \Omega^{2}\right)$, such that $\partial \Omega=\partial^{\prime} \cup \partial^{\prime \prime}$. By (2.3), there exists an open set $W^{\prime}$ containing $\partial^{\prime}$ and an integer $n_{0}^{\prime}$, such that for any $n \geqslant n_{0}^{\prime}$, the Green functions $g^{\Omega_{n}^{1}}$ can be extended as harmonic functions to $W^{\prime}$, and $\lg ^{\Omega(n}\left(z, z_{0}\right) \mid \leqslant 1$ for all $z \in W$ and $n \geqslant$ $n_{0}^{\prime}$. This follows from (2.3) since $\partial^{\prime}$, in general, will be a finite union of compact sets, each contained in an open analytic arc of $\partial \Omega^{1} \cap \partial \Omega$. Similarly we can find an open set $W^{\prime \prime}$ containing $\partial^{\prime \prime}$, and an integer $n_{0}^{\prime \prime}$, with the same properties as $W^{\prime}$ and $n_{0}^{\prime}$, but with respect to the Green functions $g^{\Omega_{n}^{2}}\left(z, z_{0}\right)$ instead.

Since $\partial^{\prime} \cup \partial^{\prime \prime}=\partial \Omega$, we can choose $W^{\prime}$ and $W^{\prime \prime}$ such that $W^{\prime} \cap \Omega \subset \Omega^{1}$ and $W^{\prime \prime} \cap \Omega \subset \Omega^{2}$.

The extensions of $g^{\Omega_{n}^{1}}\left(z, z_{0}\right)$ to $W^{\prime}$, which we also denote by $g^{\Omega_{n}^{1}}\left(z, z_{0}\right)$, are uniformly bounded in $W^{\prime}$, for $n \geqslant n_{0}^{\prime}$. Therefore there exists a subsequence $g^{\Omega n_{k}}$, converging uniformly on compact subsets of $W^{\prime}$ to a harmonic function $h$. Since for any $z \in \Omega^{1}, g^{\Omega_{n}^{1}}\left(z, z_{0}\right)$ increases to $g^{\Omega^{1}}\left(z, z_{0}\right)$, we must have $h(z)=$ 
$g^{\Omega^{1}}\left(z, z_{0}\right)$, in $\Omega^{1} \cap W^{\prime}$, and hence, for all $z \in W^{\prime}$ (recall that we can extend $g^{\Omega^{1}}\left(z, z_{0}\right)$ to $\left.W^{\prime}\right)$.

Since any convergent subsequence of $g^{\Omega_{n}^{1}}$ converges to $g^{\Omega^{1}}$, and since the functions $g^{\Omega_{n}^{1}}$ are uniformly bounded in $W^{\prime}$, it follows that $\lim _{n \rightarrow \infty} g^{\Omega_{n}^{1}}\left(z, z_{0}\right)=g^{\Omega^{1}}\left(z, z_{0}\right)$ uniformly for $z$ in compact subsets of $W^{\prime}$.

Let $U^{\prime}$ and $U^{\prime \prime}$ be two relatively compact open subsets of $W^{\prime}$ and $W^{\prime \prime}$ respectively, such that

(i) $\partial^{\prime} \subset U^{\prime}$ and $\partial^{\prime \prime} \subset U^{\prime \prime}$.

(ii) $\left|\nabla g^{\Omega^{1}}\left(z, z_{0}\right)\right|>0$ for all $z \in \bar{U}^{\prime}$, and $\left|\nabla g^{\Omega^{2}}\left(z, z_{0}\right)\right|>0$ for all $z \in \bar{U}^{\prime \prime}$.

Since $\lim _{n \rightarrow \infty} g^{\Omega_{n}^{1}}\left(z, z_{0}\right)=g^{\Omega^{1}}\left(z, z_{0}\right)$ uniformly on compact subsets of $W^{\prime}$, we will have the same type of convergence for the gradients. Thus if $m^{\prime}=$ $\inf _{z \in \partial^{\prime}}\left|\nabla_{g}^{\Omega^{1}}\left(z, z_{0}\right)\right|$, for all sufficiently large $n$ :

$$
\inf _{z \in U^{\prime}}\left|\nabla g^{\Omega_{n}^{1}}\left(z, z_{0}\right)\right| \geqslant m^{\prime} / 2>0 .
$$

Similarly, if $m^{\prime \prime}=\inf _{z \in \partial "}\left|\nabla g^{\Omega^{2}}\left(z, z_{0}\right)\right|$, for all sufficiently large $n$ :

$$
\inf _{z \in U^{\prime \prime}}\left|\nabla g^{\Omega_{n}^{2}}\left(z, z_{0}\right)\right| \geqslant m^{\prime \prime} / 2>0 .
$$

We next observe that $g^{\Omega} n\left(z, z_{0}\right)=g^{\Omega}\left(z, z_{0}\right)-1 / n$. Hence $\nabla g^{\Omega} n\left(z, z_{0}\right)=$ $\nabla g^{\Omega}\left(z, z_{0}\right)$. So if $M=\sup _{z \in \partial \Omega}\left|\nabla g^{\Omega}\left(z, z_{0}\right)\right|$, for sufficiently large values of $n$ :

$$
\sup _{z \in \partial \Omega_{n}}\left|\nabla g^{\Omega_{n}^{2}}\left(z, z_{0}\right)\right| \leqslant 2 M \text {. }
$$

Now choose $n_{0}$, so that for all $n \geqslant n_{0}(2.4 .1),(2.4 .2),(2.4 .3),(2.4 .4)$ hold, and such that $\partial \Omega_{n} \cap\left(U^{\prime} \cup U^{\prime \prime}\right)=\partial \Omega_{n}$.

For each $n \geqslant n_{0}$, let $\partial_{n}^{\prime} \subset^{\text {compact }} U^{\prime} \cap \partial \Omega_{n}^{1}$ and $\partial_{n}^{\prime \prime} \subset^{\text {compact }} U^{\prime \prime} \cap \partial \Omega_{n}^{2}$, with $\partial_{n}^{\prime} \cup \partial_{n}^{\prime \prime}=\partial \Omega_{n}$. Then by (2.4.1) we have, letting $m=\min \left(m^{\prime}, m^{\prime \prime}\right)$, for all $n \geqslant n_{0}$ :

$$
u_{\phi}^{\Omega} n\left(z_{0}\right) \leqslant(4 M / m)\left[u_{\phi}^{\Omega_{n}^{1}}\left(z_{0}\right)+u_{\phi}^{\Omega_{n}^{2}}\left(z_{0}\right)\right]
$$

If $u_{\phi}^{\Omega^{1}}$ and $u_{\phi}^{\Omega^{2}}$ exist, taking limits on both sides of (2.4.5), we will have

$$
u_{\phi}^{\Omega}\left(z_{0}\right) \leqslant(4 M / m)\left[u_{\phi}^{\Omega^{1}}\left(z_{0}\right)+u_{\phi}^{\Omega^{2}}\left(z_{0}\right)\right]<\infty
$$

proving the existence of $u_{\phi}^{\Omega}$.

REMARKS. In (2.2) and (2.4), we assumed that $\partial \Omega$ consisted of separated analytic closea curves. If instead $\partial \Omega$ is nice (that is, finitely many disjoint closed Jordan curves, each composed of finitely many analytic arcs), and $\left\{\Omega, \Omega^{1}, \Omega^{2}\right\}$ 
satisfies all other requirements for (2.4), we can map $\Omega$ one-to-one conformally onto a domain whose boundary consists of closed analytic curves. Let $\chi$ be the conformal map, then $\chi$ extends to a homeomorphism between the closure of $\Omega$ and $\chi(\Omega)$, and the triple $\left\{\chi(\Omega), \chi\left(\Omega^{1}\right), \chi\left(\Omega^{2}\right)\right\}$ will satisfy all requirements for (2.4). Thus we have

(2.5) Corollary. Let $\Omega$ be a bounded domain with nice boundary. Let $\Omega^{1}, \Omega^{2}$ be two subdomains of $\Omega$ with nice boundaries, such that

(i) $\Omega=\Omega^{1} \cup \Omega^{2}$,

(ii) $\partial \Omega=\operatorname{int}\left(\partial \Omega \cap \partial \Omega^{1}\right) \cup \operatorname{int}\left(\partial \Omega \cap \partial \Omega^{2}\right)$.

Let $z_{0} \in \Omega^{1} \cap \Omega^{2}$. There is a constant $A>0$ such that if $\phi$ is a positive subharmonic function with least harmonic majorants $u_{\phi}^{\Omega^{1}}$ and $u_{\phi}^{\Omega^{2}}$, in $\Omega^{1}$ and $\Omega^{2}$ respectively, then it has a least harmonic majorant $u_{\phi}^{\Omega}$ in $\Omega$, and $u_{\phi}^{\Omega}\left(z_{0}\right) \leqslant$ $A\left[u_{\phi}^{\Omega^{1}}\left(z_{0}\right)+u_{\phi}^{\Omega^{2}}\left(z_{0}\right)\right]$.

(2.6) Definition. Let $O$ be an open set in $\mathbf{C}^{\boldsymbol{n}}$. A function $\phi$ defined on 0 is said to be $n$-subharmonic if

(i) $\phi$ is upper semicontinuous.

(ii) $\phi$ is subharmonic in each variable separately. A function $h$ in 0 is said to be $n$-harmonic if:

(i) $h$ is continuous.

(ii) $h$ is harmonic in each variable separately.

Suppose that $\Omega^{\alpha}, \Omega^{\beta}, \cdots, \Omega^{\delta}$ are $n$ domains in $\mathrm{C}$, with nice boundaries. If $\phi$ is an $n$-subharmonic function in a region containing $\bar{\Omega}^{\alpha} \times \bar{\Omega}^{\beta} \times \cdots \times \bar{\Omega}^{\delta}$, its least $n$-harmonic majorant is given by

$$
\begin{array}{r}
u_{\phi}\left(z_{1}, \cdots, z_{n}\right)=\int_{\partial \Omega^{\alpha}} \cdots \int_{\partial \Omega^{\delta}} \phi\left(\omega_{1}, \cdots, \omega_{n}\right)\left|\nabla g^{\Omega^{\alpha}}\left(z_{1}, \omega_{1}\right)\right| \cdots \\
\left|\nabla g^{\Omega^{\delta}}\left(z_{n}, \omega_{n}\right)\right| d m\left(\omega_{1}\right) \cdots d m\left(\omega_{n}\right) .
\end{array}
$$

If $\left\{\Omega_{k}^{\alpha}\right\}, \cdots,\left\{\Omega_{k}^{\delta}\right\}$ are sequences of domains with nice boundaries, increasing to $\Omega^{\alpha}, \cdots, \Omega^{\delta}$ respectively, and $\phi$ is an $n$-subharmonic function in $\Omega^{\alpha} X$ $\cdots \times \Omega^{\delta}$, with $n$-harmonic majorants $u_{\phi, k}$ in each polydomain $\Omega_{k}^{\alpha} \times \cdots$ $\mathrm{X} \Omega_{k}^{\delta}$, then $u_{\phi, k}$ is an increasing sequence of $n$-harmonic functions which either converges to the least $n$-harmonic majorants of $\phi$ in $\Omega^{\alpha} \times \cdots \times \Omega^{\delta}$, if it exists, or diverges otherwise at every point.

(2.7) LEMmA. Let $\Omega$ be a domain in $\mathbf{C}$ and $\Omega^{1}, \Omega^{2}$, be two subdomains of $\Omega$, such that $\left\{\Omega, \Omega^{1}, \Omega^{2}\right\}$ satisfy the hypothesis of $(2.5)$. Let $\Omega^{\beta}, \cdots, \Omega^{\delta}$ be domains in $\mathrm{C}$ with nice boundaries, and $\phi$ be a positive $n$-subharmonic function in $\Omega \times \Omega^{\beta} \times \cdots \times \Omega^{\delta}$ with $n$-harmonic majorants $h_{1}$ in $\Omega^{1} \times \Omega^{\beta} \times \cdots \times \Omega^{\delta}$, and $h_{2}$ in $\Omega^{2} \times \Omega^{\beta} \times \cdots \times \Omega^{\delta}$. Then $\phi$ has an $n$-harmonic majorant in $\Omega \times$ $\Omega^{\beta} \times \cdots \times \Omega^{\delta}$. 
Proof. (2.5) is the particular case $n=1$ of the above.

Suppose $n \geqslant 2$. Fix $\left(z_{1}^{0}, z_{2}^{0}, \cdots, z_{n}^{0}\right) \in\left(\Omega^{1} \cap \Omega^{2}\right) \times \Omega^{\beta} \times \cdots \times \Omega^{\delta}$. For each $k=1,2, \cdots$ define $\Omega_{k}=\left\{z_{1} \in \Omega \mid g^{\Omega}\left(z_{1}, z_{1}^{0}\right)>1 / k\right\}$. Similarly, define $\Omega_{k}^{\beta}, \cdots, \Omega_{k}^{\delta}$; and for every $k$ let $\Phi_{k}$ be a function in $\Omega$ defined by

$$
\begin{aligned}
\Phi_{k}\left(z_{1}\right)=\int_{\partial \Omega_{k}^{\beta}} \cdots \int_{\partial \Omega_{k}^{\delta}} \phi\left(z_{1}, \omega_{2}, \cdots, \omega_{n}\right)\left|\nabla g^{\Omega^{\beta}}\left(z_{2}^{0}, \omega_{2}\right)\right| \cdots \\
\\
\left|\nabla g^{\Omega^{\delta}}\left(z_{n}^{0}, \omega_{n}\right)\right| d m\left(\omega_{1}\right) \cdots d m\left(\omega_{n}\right) .
\end{aligned}
$$

Each $\Phi_{k}$ is positive and subharmonic in $\Omega$. Moreover, if $z_{1} \in \Omega^{1}$, $\Phi_{k}\left(z_{1}\right) \leqslant h_{1}\left(z_{1}, z_{2}^{0}, \cdots, z_{n}^{0}\right)$, and if $z_{1} \in \Omega^{2}, \Phi_{k}\left(z_{1}\right) \leqslant h_{2}\left(z_{1}, z_{2}^{0}, \cdots, z_{n}^{0}\right)$, for every $k=1,2, \cdots$. Therefore, by (2.5), there exists a constant $A>0$, such that for every $k$ :

$$
\begin{aligned}
\int_{\partial \Omega_{k}} \Phi_{k}\left(\omega_{1}\right)\left|\nabla g^{\Omega}\left(z_{1}^{0}, \omega_{1}\right)\right| d m\left(\omega_{1}\right) \\
\quad \leqslant \lim _{j \rightarrow \infty} \int_{\partial \Omega_{j}} \Phi_{k}\left(\omega_{1}\right)\left|\nabla g^{\Omega}\left(z_{1}^{0}, \omega_{1}\right)\right| d m\left(\omega_{1}\right) \\
\leqslant A\left[h_{1}\left(z_{1}^{0}, \cdots, z_{n}^{0}\right)+h_{2}\left(z_{1}^{0}, \cdots, z_{n}^{0}\right)\right] .
\end{aligned}
$$

Therefore

$$
\begin{aligned}
& \lim _{k \rightarrow \infty} \int_{\partial \Omega_{k}} \cdots \int_{\partial \Omega_{k}^{\delta}} \phi\left(\omega_{1}, \cdots, \omega_{n}\right)\left|\nabla g^{\Omega_{k}}\left(z_{1}, \omega_{1}\right)\right| \cdots \\
& \left|\nabla g^{\Omega_{k}^{\delta}}\left(z_{n}, \omega_{n}\right)\right| d m\left(\omega_{1}\right) \cdots d m\left(\omega_{n}\right)
\end{aligned}
$$

is finite, and $\phi$ has an $n$-harmonic majorant in $\Omega \times \Omega^{\beta} \times \cdots \times \Omega^{\delta}$.

(2.8) Definition. Let $\phi$ be an $n$-harmonic function in some open set $0 \subset \mathrm{C}^{n}, n \geqslant 1$. We say that $\phi$ has local $n$-harmonic majorants if there is an open covering $\left\{O_{j}\right\}_{j \in I}$ of $\bar{O}$, and on each set $O_{j} \cap 0$ an $n$-harmonic majorant $h_{j}$ of $\phi$.

Our aim is to show that for certain classes of product domains (domains if $n=1$ ) it is the same for an $n$-subharmonic function to have an $n$-harmonic majorant, or local $n$-harmonic majorants.

We will start by establishing a pattern for partitioning a circle domain.

(2.9) Notation. Let $\Omega$ be a bounded domain in $\mathrm{C}$ whose boundary consists of separated circles. Assume that the outer circle is centered at the origin and has radius one. We associate with $\Omega$ a sequence of partitions, as follows.

The sets of the first partition are $\Omega_{1}^{1}=\{z \in \Omega: \operatorname{Re} z<\delta\}$ and $\Omega_{2}^{1}=$ $\left\{z \in \Omega: \operatorname{Re} z>-\delta_{1}\right\}$, where $0<\delta_{1}<1 / 2$ is chosen so that both $\Omega_{1}^{1}$ and 
$\Omega_{2}^{1}$ have nice boundaries. This can be done if we make sure that the lines $\operatorname{Re} z=\delta_{1}$ and $\operatorname{Re} z=-\delta_{1}$ are not tangent to any of the circles that compose the boundary of $\Omega$.

The second partition is a refinement of the first. The sets which are contained in $\Omega_{1}^{1}$ are $\Omega_{1}^{2}=\left\{z \in \Omega_{1}^{1}: \operatorname{Im} z<\delta_{2}\right\}$ and $\Omega_{2}^{2}=\left\{z \in \Omega_{1}^{1}: \operatorname{Im} z>-\delta_{2}\right\}$, where $\delta_{2}$ is chosen so that $\Omega_{1}^{2}$ and $\Omega_{2}^{2}$ have nice boundaries. In an analogous way we define $\Omega_{3}^{2}$ and $\Omega_{4}^{2}$, as the sets of the second partition which are contained in $\Omega_{2}^{1}$.
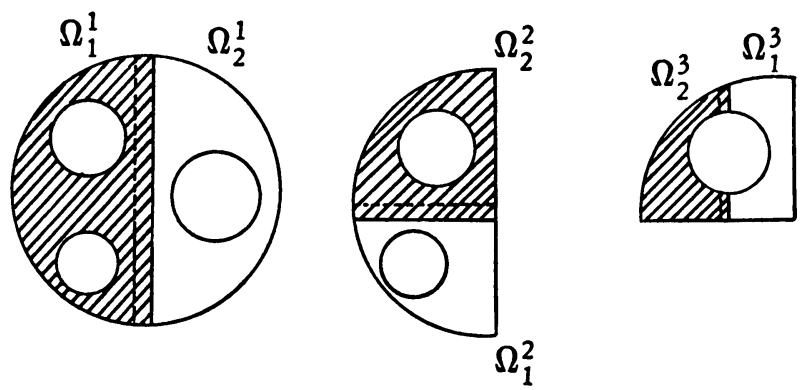

In this manner, dividing alternatively with respect to the real and imaginary parts, we have a sequence of partitions for $\Omega$. The composing sets are connected open sets with nice boundaries. Also the diameters of the sets of the $n$th partition approach zero as $n$ gets large.

(2.10) THEOREM. Let $\Omega_{\alpha}, \Omega_{\beta}, \cdots, \Omega_{\delta}$ be $n$ bounded domains in $\mathbf{C}$, whose boundaries consist of finitely many separated nondegenerate Jordan curves. Then any positive n-subharmonic function $\phi$ in $\Omega_{\alpha} \times \Omega_{\beta} \times \cdots \times \Omega_{\delta}$ with local n-harmonic majorants has an $n$-harmonic majorant.

Proof. We can assume that each domain $\Omega_{\alpha}, \cdots, \Omega_{\delta}$ is bounded by circles and that the outer circles have radius one and are centered at the origin (circle normalization theorem).

Suppose $\phi$ does not have an $n$-harmonic majorant in $\Omega_{\alpha} \times \cdots \times \Omega_{\delta}$. Decompose $\Omega_{\alpha}$ as in (2.9). Then by (2.8) $\phi$ cannot have $n$-harmonic majorants on both $\Omega_{\alpha^{1}}^{1} \times \Omega_{\beta} \times \cdots \times \Omega_{\delta}$ and $\Omega_{\alpha^{2}}^{1} \times \Omega_{\beta} \times \cdots \times \Omega_{\delta}$. Decomposing $\Omega_{\beta}$ as $\Omega_{\alpha}$, we cannot have $n$-harmonic majorants on both $\Omega_{\alpha^{1}}^{1} \times$ $\Omega_{\beta^{1}}^{1} \times \cdots \times \Omega_{\delta}$ and $\Omega_{\alpha^{1}}^{1} \times \Omega_{\beta^{2}}^{1} \times \cdots \times \Omega_{\delta}$. Choose the polydomain where $\phi$ does not have an $n$-harmonic majorant, and continue this process. The diameter of the polydomains on which there is no $n$-harmonic majorant eventually decreases to zero. But there is an open covering $\left\{O_{j}\right\}$ of $\bar{\Omega}_{\alpha} \times \bar{\Omega}_{\beta} \times \cdots \times \bar{\Omega}_{\delta}$, such that on each $O_{j} \cap\left(\Omega_{\alpha} \times \cdots \times \Omega_{\delta}\right) \phi$ has an $n$-harmonic majorant. One of the polydomains on which $\phi$ has no $n$-harmonic majorant will fall inside some 
$\mathrm{O}_{j} \cap\left(\Omega_{\alpha} \times \cdots \times \Omega_{\delta}\right)$, which is a contradiction. Therefore $\phi$ must have an $n$-harmonic majorant.

III. The multiplicative Cousin problem for $N\left(U^{n}\right)$.

(3.1) Notation AND Definitions. The field of complex numbers will be denoted by $\mathbf{C}$, and the domain of integers by $\mathbf{Z}$. $U$ will be the open unit disc and $T$ its boundary.

For any positive integer $n$, and any set $S \subset \mathrm{C}, S^{n}$ will be the cartesian product of $n$ copies of $S$. A point in $\mathbf{C}^{n}$ will be denoted by $\left(z_{1}, z_{2}, \cdots\right.$, $\left.z_{n}\right)$, and sometimes by $z=\left(z_{1}, z^{\prime}\right)$, where $z^{\prime}=\left(z_{2}, \cdots, z_{n}\right) . \mathrm{C}^{n}$ will be endowed with the usual algebraic and topological structures.

Let $z \in U^{n}$ and $\omega \in T^{n}$, then the $n$-dimensional Poisson kernel

$$
\left(\frac{1-\left|z_{1}\right|^{2}}{\left|1-z_{1} \bar{\omega}_{1}\right|^{2}}\right) \cdots\left(\frac{1-\left|z_{n}\right|^{2}}{\left|\dot{1}-z_{n} \bar{\omega}_{n}\right|^{2}}\right)
$$

will be denoted by $P(z, \omega)$. The class of complex Borel measures on $T^{n}$ will be denoted by $M\left(T^{n}\right)$, and $m_{n}$ will represent the Haar measure on $T^{n}$.

If $\mu \in M\left(T^{n}\right)$ and $f(z)=\int_{T^{n}} P(z, \omega) d \mu(\omega)$, where $z \in U^{n}, f$ will be called the Poisson integral of $\mu$ and represented by $P[d \mu]$.

Let $\Omega$ be an open set in $\mathbf{C}^{n}$. $H(\Omega)$ will be the class of holomorphic functions in $\Omega, H^{\infty}(\Omega)$ the class of bounded holomorphic functions in $\Omega$, $N(\Omega)$ the class of functions $f \in H(\Omega)$ such that $\log +|f|$ has an $n$-harmonic majorant, and $h^{1}(\Omega)$ the class of functions $f \in H(\Omega)$ such that $|\operatorname{Re} f|$ (the absolute value of its real part) has an $n$-harmonic majorant. If $\Omega$ is the polydisc $U^{n}, N\left(U^{n}\right)$ can be characterized as the class of holomorphic functions $f$ in $U^{n}$ for which

$$
\sup _{0 \leqslant r<1} \int_{T^{n}} \log ^{+}|f(r \omega)| d m_{n}(\omega)<\infty,
$$

and $h^{1}\left(U^{n}\right)$ as the class of holomorphic functions in $U^{n}$ whose real parts are Poisson integrals of measures in $M\left(T^{n}\right)$.

We say that $F$ is locally in $N(\Omega)$ if there exists an open covering $\left\{\Omega_{\alpha}\right\}$ of $\bar{\Omega}$ such that for each $\alpha, f$ restricted to $\Omega_{\alpha} \cap \Omega$ belongs to $N\left(\Omega_{\alpha} \cap \Omega\right)$. The class of functions locally in $N(\Omega)$ will be denoted by $N_{\text {LOC }}(\Omega)$. Similarly we define $h_{\text {LOC }}^{1}(\Omega)$. Observe that both $|\operatorname{Re} f|$. and $\log ^{+}|f|$ are $n$-subharmonic. From (2.10), it follows for a suitable polydomain $\Omega$, that

$$
\begin{aligned}
& N_{\text {LOC }}(\Omega)=N(\Omega), \\
& h_{\text {LOC }}^{1}(\Omega)=h^{1}(\Omega) .
\end{aligned}
$$

The class $N_{\text {LOC }}(\Omega)$ has the structure of an algebra. We denote the group of its invertible elements by inv $N_{\text {LOC }}(\Omega)$. It is easy to see that if $\Omega$ is 
simply connected, then $f \in \operatorname{inv} N_{\text {LOC }}(\Omega)$ if and only if $f=\exp g$ with $g \in$ $h_{\text {LOC }}^{1}(\Omega)$. Clearly, $h^{1}(\Omega)$ has the structure of a vector space.

REMARK. For a class of holomorphic functions in an open set $\Omega \subset \mathbf{C}^{n}$, invariance under unitary maps is desirable. This will not be the case with the classes defined above unless $\Omega$ is a polydomain. Except for a few instances, these classes will be considered only over polydomains.

All other notation introduced in this section will be standard.

The following lemma closely resembles Lemma 1.2 of [6].

(3.2) Lemma. Let $f \in h^{1}\left(U^{n}\right)$, and let $\lambda_{1}$ and $\lambda_{2}$ be disjoint arcs in $T$. Define $V_{j}, j=1,2$, to be the union of $U$, the interior of $\lambda_{j}$, and the exterior of $\bar{U}$ in the Riemann sphere $S^{2}$.

Then, for all $z \in U^{n}$, we may write $f(z)=f_{1}(z)+f_{2}(z)$, where $f_{j} \in$ $H\left(V_{j} \times U^{n-1}\right), f_{j} \in h^{1}\left(U^{n}\right), f_{j} \in h^{1}\left(\left(S^{2}-\bar{U}\right) \times U^{n-1}\right)$, and $f_{j} \in h^{1}\left(\Omega_{j} \times U^{n-1}\right)$ for some open set $\Omega_{j}$ in $\mathrm{C}$ containing $\lambda_{j}$.

Proof. Let $E=\left\{k \in Z^{n}: k_{1} \geqslant 0, \cdots, k_{n} \geqslant 0\right.$, or $\left.k_{1} \leqslant 0, \cdots, k_{n} \leqslant 0\right\}$.

Given $k \in \mathbf{Z}^{n}$, let $E^{+}(k)=\left\{j \in \mathbf{Z}^{n}: k_{1} \leqslant j_{1}, \cdots, k_{n} \leqslant j_{n}\right\}$ and $E^{-}(k)=\left\{j \in \mathbf{Z}: k_{1} \geqslant j_{1}, \cdots, k_{n} \geqslant j_{n}\right\}$.

Let $z \in U^{n}$ and $\omega \in T^{n}$; the series expansion for the Poisson kernel is

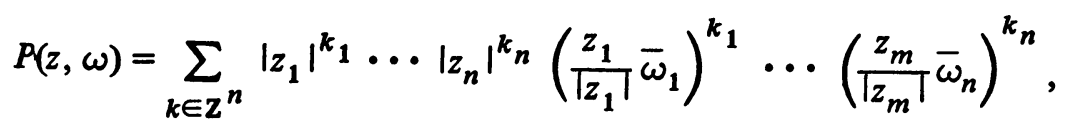

and we define

$$
K(z, \omega)=\sum_{k \in E}\left|z_{1}\right|^{k_{1}} \cdots\left|z_{n}\right|^{k_{n}}\left(\frac{z_{1}}{\left|z_{1}\right|} \bar{\omega}_{1}\right)^{k_{1}} \cdots\left(\frac{z_{n}}{\left|z_{n}\right|} \bar{\omega}_{n}\right)^{k_{n}}
$$

It can be seen that

$$
K(z, \omega)=\operatorname{Re}\left\{\frac{2}{\left(1-z_{1} \bar{\omega}_{1}\right) \cdots\left(1-z_{n} \bar{\omega}_{n}\right)}-1\right\} .
$$

Let $f$ be the given function, and write it as $f=f^{\prime}+f^{\prime \prime}$, where $f^{\prime}\left(z_{1}, z_{2}, \cdots, z_{n}\right)=f\left(0, z_{2}, \cdots, z_{n}\right)$ and $f^{\prime \prime}=f-f^{\prime}$. The function $f^{\prime}$ is clearly in $h^{1}\left(S^{2} \times U^{n-1}\right)$, so it suffices to prove the lemma with $f$ replaced by $f^{\prime \prime}$.

Let $f^{\prime \prime}=u+i v$. Since $f^{\prime \prime} \in h^{1}\left(U^{n}\right)$, there is a measure $\mu \in M\left(T^{n}\right)$ such that $u=P[d \mu]$. So if $0 \leqslant \rho_{1}, \cdots, \rho_{n}<1$, we may write

$$
u\left(\rho_{1} e^{i \theta_{1}}, \cdots, \rho_{n} e^{i \theta_{n}}\right)=\sum_{k \in E} \hat{\mu}(k) \rho_{1}^{\left|k_{1}\right|} \cdots \rho_{n}^{\left|k_{n}\right|} e^{i k_{1} \theta_{1}} \cdots e^{i k_{n} \theta_{n}}
$$

But $f^{\prime \prime}\left(0, z_{2}, \cdots, z_{n}\right)=0$, and $u=\operatorname{Re} f^{\prime \prime}$, so we must have $\hat{\mu}\left(0, k_{2}, \cdots, k_{n}\right)$ for all choices of $k_{2}, \cdots, k_{n}$. Thus the summation in (3.2.3) 
extends over the set $E^{+}(1,0, \cdots, 0) \cup E^{-}(-1,0, \cdots, 0)$.

Let $h$ be a real function in $C^{\infty}(T)$ which vanishes identically in a neighborhood of $\lambda_{1}$ and is identically one in a neighborhood of $\lambda_{2}$. Then $h\left(\omega_{1}\right)=$ $\Sigma_{-\infty}^{+\infty} c_{m} \omega_{1}^{m}, \bar{c}_{m}=c_{-m}$, and $\left|c_{m}\right|=O\left(m^{-2}\right)$.

Define

$$
g_{1}(z)=\int_{T^{n}} h\left(\omega_{1}\right)\left[\frac{2}{\left(1-\bar{\omega}_{1} z_{1}\right) \cdots\left(1-\bar{\omega}_{n} z_{n}\right)}-1\right] d \mu(\omega),
$$

and

$$
g_{2}(z)=\int_{T^{n}}\left(1-h\left(\omega_{1}\right)\right)\left[\frac{2}{\left(1-\bar{\omega}_{1} z_{1}\right) \cdots\left(1-\bar{\omega}_{n_{n}} z\right)}-1\right] d \mu(\omega) .
$$

Clearly each $g_{j}$ is holomorphic in $V_{j} \times U^{n-1}$, and for some imaginary constant $\gamma, f^{\prime \prime}=g_{1}+g_{2}+\gamma$.

Proof that $g_{1} \in h^{1}\left(U^{n}\right)$. Let $z \in U^{n}$, then

$$
\begin{aligned}
\operatorname{Re} g_{1}(z)= & \int_{T^{n}} h\left(\omega_{1}\right) k(z, \omega) d \mu(\omega) \\
= & c_{0} \int_{T^{n}} k(z, \omega) d \mu(\omega) \\
& \quad+\sum_{m=1}^{\infty} \int_{T^{n}}\left[c_{m} \omega_{1}^{m}+\bar{c}_{m} \bar{\omega}_{1}^{m}\right] k(z, \omega) d \mu(\omega) .
\end{aligned}
$$

Let $\mu_{m}$ and $\nu_{m}$ be measures in $M\left(T^{n}\right)$ such that the Fourier transform $\hat{\mu}_{m}$ is the characteristic function of $\left\{k \in \mathbf{Z}^{n}:-m+1 \leqslant k_{1} \leqslant-1\right\}$ and $\hat{v}_{m}$ is the characteristic function of $\left\{k \in \mathrm{Z}^{n}: 1 \leqslant k_{1} \leqslant m-1\right\}$. Both $\mu_{1}$ and $\nu_{1}$ are the zero measures.

For some absolute constant $C, \mu_{m}$ and $\nu_{m}$ have norms no larger than $C \log (1+m)$.

Let $A_{m}$, for each $m=1,2, \cdots$, be a measure on $T^{n}$ defined by $d A_{m}=c_{m} \omega_{1}^{m}\left[d \mu-d\left(\mu_{*} \mu_{m}\right)\right]+\bar{c}_{m} \bar{\omega}_{1}^{m}\left[d \mu-d\left(\mu_{*} \nu_{m}\right)\right]$.

If $\left\|A_{m}\right\|$ is its total variation, we have

$$
\left\|A_{m}\right\| \leqslant 2 C\|\mu\|\left|c_{m}\right|(1+\log (1+m))
$$

for every $m=1,2, \cdots$. Thus $\Sigma_{m=1}^{\infty}\left\|A_{m}\right\|<\infty$, so $\sum_{m=1}^{\infty} A_{m}$ is a measure in $M\left(T^{n}\right)$.

Since $\int_{T^{n}}\left[c_{m} \omega_{1}^{m}+\bar{c}_{m} \bar{\omega}_{1}^{m}\right] k(z, \omega) d \mu(\omega)=\int_{T^{n}} P(z, \omega) d A_{m}(\omega)$, substituting in (3.2.4) we have $\operatorname{Re} g_{1}(z)=\int_{T^{n}} P(z, \omega) d \sigma(\omega)$, where $\sigma=c_{0} \mu+$ $\sum_{m=1}^{\infty} A_{m}$. Being $\operatorname{Re} g_{1}$ the Poisson integral of a measure, it follows that

$$
g_{1} \in h^{1}\left(U^{n}\right)
$$


Proof that $g_{1} \in h^{1}\left(\left(S^{2}-U\right) \times U^{n-1}\right)$. Let $z \in U^{n}$. Then

$$
\begin{aligned}
& g_{1}\left(z_{1}, z^{\prime}\right)-g\left(1 / \bar{z}_{1}, z^{\prime}\right) \\
& \quad=\int_{T^{n}} h\left(\omega_{1}\right) P\left(z_{1}, \omega_{1}\right) \frac{2}{\left(1-\bar{\omega}_{1} z_{1}\right) \cdots\left(1-\bar{\omega}_{n} z\right)} d \mu(\omega) .
\end{aligned}
$$

Taking real parts:

$$
\begin{aligned}
\operatorname{Re} g_{1}\left(z_{1}, z^{\prime}\right) & -\operatorname{Re} g_{1}\left(1 / \bar{z}_{1}, z^{\prime}\right) \\
& =\int_{T^{n}} h\left(\omega_{1}\right) P\left(z_{1}, \omega_{1}\right)\left(1+K\left(z^{\prime}, \omega^{\prime}\right)\right) d \mu(\omega) .
\end{aligned}
$$

But $\hat{\mu}$ vanishes off $E$, so the second term above equals

$$
\int_{T^{n}} h\left(\omega_{1}\right) P\left(z_{1}, \omega_{1}\right)\left(1+P\left(z^{\prime}, \omega^{\prime}\right)\right) d \mu(\omega)
$$

If we take absolute values on both sides of (3.2.6), we then obtain

$$
\begin{aligned}
\mid \operatorname{Re} g_{1}\left(z_{1}, z^{\prime}\right) & -\operatorname{Re} g_{1}\left(\frac{1}{\bar{z}_{1}}, z^{\prime}\right) \mid \\
& \leqslant \int_{T^{n}}\left|h\left(\omega_{1}\right)\right| P\left(z_{1}, \omega_{1}\right)\left(1+P\left(z^{\prime}, \omega^{\prime}\right)\right) d|\mu|(\omega) .
\end{aligned}
$$

The last term above is $n$-harmonic, and since $g_{1} \in h^{1}\left(U^{n}\right)$, it follows that $\left|\operatorname{Re} g_{1}\left(1 / \bar{z}_{1}, z^{\prime}\right)\right|$ has an $n$-harmonic majorant in $U^{n}$ which we will denote by $\phi$.

Suppose now that

$$
z=\left(z_{1}, z^{\prime}\right) \in\left(\left(S^{2}-\bar{U}\right) \times U^{n-1}\right) ;
$$

then $\left|\operatorname{Re} g_{1}\left(z_{1}, z^{\prime}\right)\right| \leqslant \phi\left(1 / \bar{z}, z^{\prime}\right)$. Since $\phi\left(1 / \overline{z_{1}}, z^{\prime}\right)$ is $n$-harmonic in $\left(S^{2}-\bar{U}\right) \times$ $U^{n-1}$, it follows that:

$$
g_{1} \in h^{1}\left(\left(S^{2}-\bar{U}\right) \times U^{n-1}\right)
$$

Proof that $g_{1} \in h^{1}\left(\Omega_{1} \times U^{n-1}\right)$. The function $h$ was chosen so that it vanishes identically in some neighborhood $\Gamma$ of $\lambda_{1}$. With center at some point of $\lambda_{1}$, take a circle $C$, such that the portion of $T$ enclosed by $C$ contains $\lambda_{1}$, and is contained in $\Gamma$. We also impose on $C$ the following conditions: if $\xi^{\prime}, \xi^{\prime \prime}$ are the points where $C$ intersects $T, h_{U}$ is the $n$-harmonic majorant of $\left|\operatorname{Re} g_{1}\right|$ in $U^{n}$, and $h_{S^{2}-\bar{U}}$ that of $\left|\operatorname{Re} g_{1}\right|$ in $\left(S^{2}-\bar{U}\right) X$ $U^{n-1}$, then the nontangential limit of $h_{U}\left(z_{1}, 0, \cdots, 0\right)$ and of $h_{S^{2}-U}\left(z_{1}, 0, \cdots, 0\right)$ exist as $z_{1}$ approaches $\xi^{\prime}$ or $\xi^{\prime \prime}$. 


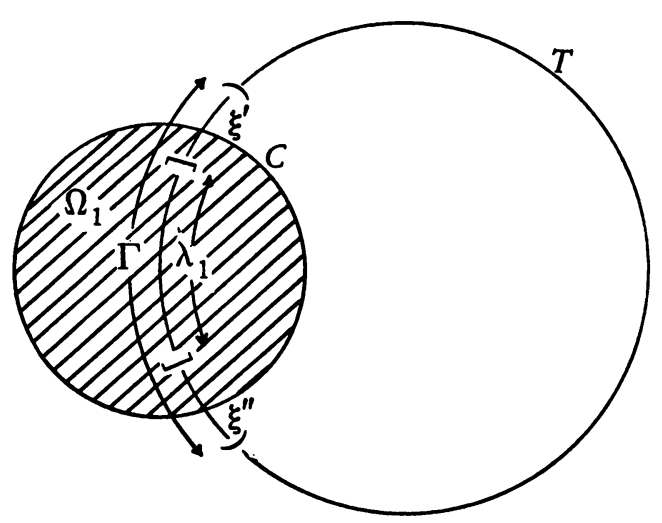

Let $\Omega_{1}$ be the disc bounded by $C$, and let $W=U \cup\left(S^{2}-\bar{U}\right) \cup \Gamma$. It can be seen, by its definition, that $g_{1} \in H\left(W \times U^{n-1}\right)$. Our goal then is to prove that $\left|\operatorname{Re} g_{1}\right|$ has an $n$-harmonic majorant in $\Omega_{1} \times U^{n-1}$.

If $0<r<1$, and $\left(z_{1}, z^{\prime}\right) \in \Omega_{1} \times U^{n-1}$, we have

$$
\operatorname{Re} g_{1}\left(z_{1}, r z^{\prime}\right)
$$

$$
=\int_{C} \int_{T^{n-1}} \operatorname{Re} g_{1}\left(\xi, r \omega^{\prime}\right) P_{C}\left(z_{1}, \xi\right) P\left(z^{\prime}, \omega^{\prime}\right) d m_{n-1}\left(\omega^{\prime}\right) d m_{1}(\xi)
$$

where $P_{C}\left(z_{1}, \xi\right)$ is the one-dimensional Poisson kernel for the disc $\Omega_{1}$ and $m_{1}$ is the Haar measure on $C$. This follows because $\operatorname{Re} g_{1}\left(z_{1}, r z^{\prime}\right)$ is a continuous $n$-harmonic function for $\left(z_{1}, z^{\prime}\right)$ in $\Omega_{1} \times U^{n-1}$, and as such must be the Poisson integral of its boundary values.

Also, if $\xi \in U$, we have $\left|\operatorname{Re} g_{1}\left(\xi, r \omega^{\prime}\right)\right| \leqslant h_{U}\left(\xi, r \omega^{\prime}\right)$, and hence:

$\int_{T^{n-1}}\left|\operatorname{Re} g_{1}\left(\xi, r \omega^{\prime}\right)\right| d m_{n-1}\left(\omega^{\prime}\right) \leqslant \int_{T^{n-1}} h_{U}\left(\xi, r \omega^{\prime}\right) d m_{n-1}\left(\omega^{\prime}\right)$

Similarly, if $\xi \in S^{2}-\bar{U}$ :

$$
=h_{U}(\xi, 0, \cdots, 0) \text {. }
$$

$$
\begin{aligned}
\int_{T^{n-1}}\left|\operatorname{Re} g_{1}\left(\xi, r \omega^{\prime}\right)\right| d m_{n-1}\left(\omega^{\prime}\right) & \leqslant \int_{T^{n-1}{ }^{n}{ }_{S^{2}-\bar{U}}\left(\xi, r \omega^{\prime}\right) d m_{n-1}\left(\omega^{\prime}\right)} \\
& =h_{S^{2}-\bar{U}}(\xi, 0, \cdots, 0) .
\end{aligned}
$$

Since the nontangential limits of both $h_{U}(\xi, 0,0, \cdots, 0)$ and of $h_{S^{2}-\bar{U}}(\xi, 0, \cdots, 0)$ exists as $\xi$ approaches either $\xi^{\prime}$ or $\xi^{\prime \prime}$, for some constant $M$, any $\xi \in C$, and any $0<r<1$, we have

$$
\int_{T^{n-1}}^{\cdot}\left|\operatorname{Re} g_{1}\left(\xi, r \omega^{\prime}\right)\right| d m_{n-1}\left(\omega^{\prime}\right)<M .
$$

Thus, for any $0<r<1$ : 


$$
\int_{C} \int_{T^{n-1}}\left|\operatorname{Re} g_{1}\left(\xi, r \omega^{\prime}\right)\right| d m_{n-1}\left(\omega^{\prime}\right) d m_{1}(\xi)<M<\infty .
$$

The above now implies that there exist a sequence $r_{k}$ increasing to 1 , and a measure $\lambda \in M\left(C \times T^{n-1}\right)$, such that the measures

$$
\operatorname{Re} g_{1}\left(\xi, r_{k} \omega^{\prime}\right) d m_{n-1}\left(\omega^{\prime}\right) d m_{1}(\xi)
$$

approach $d \lambda\left(\xi, \omega^{\prime}\right)$ weakly as $r_{k}$ approaches 1 . Thus, taking limits on both sides of (3.2.8), we obtain

$$
\operatorname{Re} g_{1}\left(z_{1}, z^{\prime}\right)=\int_{C \times T^{n-1}} P_{C}\left(z_{1}, \xi\right) P\left(z^{\prime}, \omega^{\prime}\right) d \lambda\left(\xi, \omega^{\prime}\right),
$$

for all points $\left(z_{1}, z^{\prime}\right) \in \Omega \times U^{n-1}$. Hence

$$
g_{1} \in h^{1}\left(\Omega_{1} \times U^{n-1}\right) .
$$

In an analogous way, we prove that $g_{2} \in h^{1}\left(U^{n}\right), g_{2} \in h^{1}\left(\left(S^{2}-\bar{U}\right) \times U^{n-1}\right)$ and $g_{2} \in h^{1}\left(\Omega_{2} \times U^{n-1}\right)$ for some open disc $\Omega_{2}$ containing $\lambda_{2}$. Then if we set $f_{1}=g_{1}$ and $f_{2}=g_{2}+\gamma, f^{\prime \prime}=f_{1}+f_{2}$ gives us the desired decomposition of $f^{\prime \prime}$.

For the following lemma, set:

$$
\begin{gathered}
X_{1}=\left\{z \in \mathbf{C}^{n}: z_{j}=x_{j}+i y_{j},-1 / 2<x_{1}<1 ;\right. \\
\left.\left|x_{2}\right|, \cdots,\left|x_{n}\right|,\left|y_{1}\right|, \cdots,\left|y_{n}\right|<1\right\}, \\
X_{2}=\left\{z \in \mathbf{C}^{n}: z_{j}=x_{j}+i y_{j},-1<x_{1}<1 / 2 ;\right. \\
\left.\left|x_{2}\right|, \cdots,\left|x_{n}\right|,\left|y_{1}\right|, \cdots,\left|y_{n}\right|<1\right\} .
\end{gathered}
$$

(3.3) LEMMA. If $f \in h^{1}\left(X_{1} \cap X_{2}\right)$, then we may write, for all $z \in X_{1} \cap X_{2}$, $f(z)=f_{1}(z)+f_{2}(z)$ where $f_{1} \in h^{1}\left(X_{1}\right)$ and $f_{2} \in h^{1}\left(X_{2}\right)$.

Proof. Let $\alpha$ be a conformal map from $U$ onto $\{x+i y \in \mathrm{C}:|x|<1$, $|y|<1\}$, and $\beta$ from $U$ onto $\{x+i y \in \mathrm{C}:|x|<1 / 2,|y|<1\}$. We consider $\beta$ extended to a homeomorphism between the closures of its domain and its range.

Let $\Lambda_{1}=\{1 / 2+i y:|y| \leqslant 1\}, \Lambda_{2}=\{-1 / 2+i y:|y| \leqslant 1\}$, and let $\lambda_{1}$, $\lambda_{2}$ be the preimages of $\Lambda_{1}, \Lambda_{2}$ under $\beta$. Let $V_{1}$ and $V_{2}$ be the domains of the Riemann sphere $S^{2}$ constructed from $\lambda_{1}$ and $\lambda_{2}$ as in (3.2):

$$
V_{1}=U \cup \text { int } \lambda_{1} \cup\left(S^{2}-\bar{U}\right) \text { and } V_{2}=U \cup \text { int } \lambda_{2} \cup\left(S^{2}-\bar{U}\right) \text {. }
$$

Using the reflection principle, we can extend $\beta$ to a conformal map $\beta_{1}$ from $V_{1}$ onto $\{x+i y:-1 / 2<x<1+1 / 2,|y|<1\}$ : given a point $z,|z|>$ 
1, we define $\beta_{1}(z)=1-\overline{\beta(1 / \bar{z})}$. Similarly $\beta$ can be extended to a conformal map $\beta_{2}$ from $V_{2}$ onto $\{x+i y:-1-1 / 2<x<1 / 2,|y|<1\}$.

Define ${ }^{\prime} \Phi: U^{n} \rightarrow X_{1} \cap X_{2}$ by $\Phi\left(z_{1}, z_{2}, \cdots, z_{n}\right)=\left(\beta\left(z_{1}\right), \alpha\left(z_{2}\right), \cdots\right.$ $\alpha\left(z_{n}\right)$ ), and let $\Phi_{j}$ be the extension, induced by $\beta_{j}$, of $\Phi$ to $V_{j} \times U^{n-i}$. Then the range of $\Phi_{j}$ contains $X_{j}$ for $j=1,2$.

Let $f$ be the function of our lemma, and set $G=F \circ \Phi$. Since $G \in$ $h^{1}\left(U^{n}\right)$, by (3.2)' we have $G=G_{1}+G_{2}$, where $G_{j} \in H\left(V_{j} \times U^{n-1}\right), G_{j} \in$ $h^{1}\left(U^{n}\right), G_{j} \in h^{1}\left(\left(S^{2}-\bar{U}\right) \times U^{n-1}\right)$, and $G_{j} \in h^{1}\left(\Omega_{j} \times U^{n-1}\right)$ where $\Omega_{j}$ is an open set containing $\lambda_{j}$.

If we define $f_{j}=G_{j} \circ \Phi_{j}$, on $X_{1} \cap X_{2}$ we will have $f=f_{1}+f_{2}$, where $f_{j} \in h^{1}\left(X_{1} \cap X_{2}\right), f_{j} \in h^{1}\left(X_{j}-\overline{\left.X_{1} \cap X_{2}\right)}\right.$ and $f_{j} \in h^{1}\left(\Phi_{j}^{-1}\left(\Omega_{j} \times U^{n-1}\right)\right)$. Since $\Phi_{j}^{-1}\left(\Omega_{j} \times U^{n-1}\right)=\beta_{j}^{-1}\left(\Omega_{j}\right) \times\{x+i y:|x|<1,|y|<1\}^{n-1}$, and $\beta_{j}^{-1}\left(\Omega_{j}\right)$ is the intersection of an open set containing $\Lambda_{j}$ with $X_{1} \cap X_{2}$, it follows that $f_{j}$ is locally in $h^{1}\left(X_{j}\right)$. Therefore, by (3.1.2), $f_{j} \in h^{1}\left(X_{j}\right)$, for $j=1,2$.

As a corollary to this, we have

(3.4) Corollary. If $F$ is an invertible element of $N\left(X_{1} \cap X_{2}\right)$, then on $X_{1} \cap X_{2}$ we may write $F=F_{1} F_{2}$ where $F_{j}$ is an invertible element of $N\left(X_{j}\right)$.

Proof. If both $F$ and $F^{-1}$ are in $N\left(X_{1} \cap X_{2}\right)$, then $\log ^{+}|F|$ and $\log -|F|$ have $n$-harmonic majorants in $X_{1} \cap X_{2}$. So if $F=\exp f$ and $f=$ $u+i v,|u|$ must have an $n$-harmonic majorant; i.e., $f \in h^{1}\left(X_{1} \cap X_{2}\right)$. Lemma (3.3) now asserts that $f=f_{1}+f_{2}$ where $f_{j} \in h^{1}\left(X_{j}\right)$. If we set $F_{j}=$ $\exp f_{j}, j=1,2$, then clearly each $F_{j}$ is an invertible element of $N\left(X_{j}\right)$, and $F=F_{1} F_{2}$.

Next we prove the multiplicative Cousin problem with $N$-data.

(3.5) THEOREM. Let $\left\{V_{\alpha}\right\}_{\alpha \in A}$ be an open covering of $\bar{U}^{n}$, and for each $\alpha$ let $f_{\alpha} \in N\left(V_{\alpha} \cap U^{n}\right)$. If for all $\alpha$ and $\beta, f_{\alpha} f_{\beta}^{-1}$ is an invertible element of $N\left(V_{\alpha} \cap V_{\beta} \cap U^{n}\right)$, then there exists $F \in N\left(U^{n}\right)$ such that for all $\alpha \in A, F f_{\alpha}^{-1}$ is an invertible element of $N_{\mathrm{LOC}}\left(V_{\alpha} \cap U^{n}\right)$.

REMARK. If each $V_{\alpha} \cap U^{n}$ is a polydomain as described in (2.10), then the conclusion above would be in terms of $N\left(V_{\alpha} \cap U^{n}\right)$ instead of $N_{\text {LOC }}\left(V_{\alpha} \cap U^{n}\right)$. This will be the case in the application of the result we give in the next section. Also, it is always possible to find a refinement of the given covering consisting of adequate polydomains.

Proof. It is more convenient to work in a polycube than in a polydisc. 
Let

$$
\begin{gathered}
X_{1}^{1}=\left\{z \in \mathbf{C}^{n}: z_{j}=z_{j}+i y_{j},-1<x_{1}<1 / 2 .\right. \\
\left.\left|x_{2}\right|, \cdots,\left|x_{n}\right|,\left|y_{1}\right|, \cdots,\left|y_{n}\right|<1\right\}, \\
X_{1}^{2}=\left\{z \in \mathbf{C}^{n}: z_{j}=x_{j}+i y_{j},-1 / 2<x_{1}<1,\right. \\
\left.\left|x_{2}\right|, \cdots,\left|x_{n}\right|,\left|y_{1}\right|, \cdots,\left|y_{n}\right|<1\right\},
\end{gathered}
$$

and $X=X_{1}^{1} \cup X_{1}^{2}$.

As we just mentioned, we will prove the theorem for the polycube $X$. Suppose the theorem is not true. If there were functions $F_{j} \in N\left(X_{1}^{j}\right)$ such that for every $\alpha \in A$ and $j=1,2, F_{j} f_{\alpha}^{-1}$ belonged to inv $N_{\text {LOC }}\left(X_{1}^{j} \cap V_{\alpha}\right)$, then $F_{1} F_{2}^{-1}=F_{1} f_{\alpha}^{-1} f_{\alpha} F_{2}^{-1}$ would be inv $N_{L O C}\left(X_{1}^{1} \cap X_{1}^{2} \cap V_{\alpha}\right)$ for every $\alpha$. Thus $F_{1} F_{2}^{-1}$ would be in inv $N_{\text {LOC }}\left(X_{1}^{1} \cap X_{1}^{2}\right)=\operatorname{inv} N\left(X_{1}^{1} \cap X_{1}^{2}\right)$.

Then, by (3.4) we would have $F_{2} F_{1}^{-1}=G_{2}^{-1} G_{1}$ where $G_{j} \in \operatorname{inv} N\left(X_{1}^{j}\right)$, $j=1$, 2. If we define $F=F_{1} G_{1}$ on $X_{1}^{1}, F=F_{2} G_{2}$ on $X_{1}^{2}$, it is clear that $F \in N(X)$ and that, for each $\alpha \in A, F f_{\alpha}^{-1} \in \operatorname{inv} N_{\text {LOC }}\left(V_{\alpha} \cap X\right)$. But this is not possible, since we assumed the theorem not to be true. Thus both $F_{1}$ and $F_{2}$ cannot exist. Suppose $F_{1}$ does not exist, and define

$$
X_{2}^{1}=\left\{z \in X_{1}^{1}:-1 / 2<y_{1}<1\right\}, \quad X_{2}^{2}=\left\{z \in X_{1}^{1}:-1<y_{1}<1 / 2\right\} .
$$

Arguing as before, the Cousin problem cannot be solved on both of these polycubes; so on one of the above, call it $X_{2}^{k}$, the induced problem is unsolvable.

Iterating this procedure, proceeding cyclicly through the real coordinates of $\mathrm{C}^{n}$, we obtain a nested sequence $X_{1}^{1} \supset X_{2}^{k} \supset \cdots \supset X_{m}^{k_{m}} \supset \cdots$ of polycubes with diameters eventually decreasing to zero, on none of which we are able to solve the induced problem. Since $\left\{V_{\alpha}\right\}_{\alpha \in A}$ is an open covering of $\bar{X}$, for some $\alpha \in A$ and some integer $m$ we will have $X_{m}^{k} \subset V_{\alpha}$. But then $f_{\alpha}$ solves the induced Cousin problem on $X_{m}^{k_{m}}$, a contradiction. Thus the theorem must be true.

\section{A zero set for $N\left(U^{n}\right)$.}

(4.1) Notation and definitions. The zero set of a function $f$ in $H(\Omega)$ will be denoted by $Z(f)$. Two functions $f_{1}, f_{2} \in H(\Omega)$ are said to have the same zeros if there exists a zero free holomorphic function $h \in H(\Omega)$ such that $f_{1}=f_{2} h$; thus multiplicities are taken into account.

We will say that the zero set of $f \in H(\Omega)$ is locally given by bounded holomorphic functions if there exist an open covering $\left\{V_{\alpha}\right\}$ of $\overline{Z(f)}$ in $\mathbf{C}^{n}$ and for each $\alpha$ a function $f_{\alpha} \in H^{\infty}\left(V_{\alpha} \cap \Omega\right)$ with the same zeros in $V_{\alpha} \cap$ $\Omega$ as $f$. 
Let $\Omega$ be a polydisc and $f \in H(\Omega)$. Then $Z(f)$ is said to be a Rudin variety if it is bounded away from the essential boundary of $\Omega$ (the cartesian product of the boundaries of its coordinate discs). If this is the case, Rudin proved in [5] that $f$ has the same zeros as some $F \in H^{\infty}(\Omega)$.

Finally, let $0<r<R<\infty$ and define:

$$
\begin{gathered}
U(r)=\{z \in \mathrm{C}:|z|<r\}, \quad T(r)=\{z \in \mathrm{C}:|z|=r\}, \\
Q(r, R)=\{z \in \mathrm{C}: r<|z|<R\} .
\end{gathered}
$$

We will denote $U(1)$ and $T(1)$ by $U$ and $T$ respectively.

Recall that for any set $S \subset \mathrm{C}, S^{n}$ denotes the cartesian product of $n$ copies of $S$.

(4.2) Lemma. Let $\Omega_{1}, \cdots, \Omega_{n}$ be simply connected domains in $\mathbf{C}$. Let $f_{1}$ and $f_{2}$ be bounded holomorphic functions in $\Omega_{1} \times \cdots \times \Omega_{n}$. Then if $f_{1} f_{2}^{-1}$. defines a holomorphic function in $\Omega_{1} \times \cdots \times \Omega_{n}$, it must belong to $N\left(\Omega_{1} \times \cdots \times \Omega_{n}\right)$.

Proof. There is no loss of generality if we assume that $\Omega_{j}=U$, for each $j=1, \cdots, n$, and that $\left\|f_{1}\right\|_{\infty}=\left\|f_{2}\right\|_{\infty}=1$.

Case I. $f_{2}(0, \cdots, 0) \neq 0$. Let $r<1$ and $\omega \in T^{n}$. Then

$$
\log ^{+}\left|\frac{f_{1}(r \omega)}{f(r \omega)}\right| \leqslant \log ^{+}\left|f_{1}(r \omega)\right|+\log ^{+} \frac{1}{\left|f_{2}(r \omega)\right|} \leqslant-\log \left|f_{2}(r \omega)\right| .
$$

Integrating, and applying Jensen's inequality:

$$
\int_{T^{n}} \log ^{+}\left|\frac{f_{1}(r \omega)}{f_{2}(r \omega)}\right| d m_{n}(\omega) \leqslant-\int_{T^{n}} \log \left|f_{2}(r \omega)\right| d m_{n} \leqslant-\log \left|f_{2}(0, \cdots, 0)\right| \text {. }
$$

Since the above holds for all $r<1$, it follows that $f_{1} f_{2}^{-1} \in H\left(U^{n}\right)$.

Case II. $f_{2}(0, \cdots, 0)=0$. Since $f_{2} \neq 0$, for some $\left(a_{1}, \cdots, a_{n}\right) \in$ $U^{n} f\left(a_{1}, \cdots, a_{n}\right) \neq 0$. For each $1 \leqslant j \leqslant n$ let $\chi_{j}(z)=\left(z+a_{j}\right) /\left(1+\bar{a}_{j} z\right)$. Then if $\Phi=\left(\chi_{1}, \cdots, \chi_{n}\right): U^{n} \rightarrow U^{n}$; we observe that $\left(f_{1} f_{2}^{-1}\right) \circ \Phi \in N\left(U^{n}\right)$, since it satisfies the requirement of Case I. Therefore $f_{2} f_{2}^{-1}=\left[\left(f_{1} f_{2}^{-1}\right) \circ \Phi\right]$ - $\Phi^{-1} \in N\left(U^{n}\right)$.

(4.3) Corollary. Let $\Omega_{1}, \cdots, \Omega_{n}$ be domains in C, whose boundaries consist of finitely many separated nondegenerate Jordan curves. Let $f_{1}$ and $f_{2}$ be bounded holomorphic functions in $\Omega_{1} \times \cdots \times \Omega_{n}$. Then if $f_{1} f_{2}^{-1}$ defines a holomorphic function in $\Omega_{1} \times \cdots \times \Omega_{n}$, it must belong to $N\left(\Omega_{1} \times \cdots \times \Omega_{n}\right)$.

Proof. We can decompose $\Omega_{1} \times \cdots \times \Omega_{n}$ as a finite union of prod- 
ucts of simply connected domains. By (4.2), $f_{1} f_{2}^{-1}$ is the Nevanlinna class of each one of these products. But then, $f_{1} f_{2}^{-1}$ is locally in $N\left(\Omega_{1} \times \cdots \times \Omega_{n}\right)$, therefore by (3.1.1) $f_{1} f_{2}^{-1} \in N\left(\Omega_{1} \times \cdots \times \Omega_{n}\right)$.

Suppose now that $f \in H\left(U^{n}\right)$, and that its zero set is locally given $v y$ bounded holomorphic functions. This means that there is a collection $\left\{V_{\alpha}\right\}_{\alpha \in A}$ of open sets such that $\overline{Z(f)} \subset \bigcup V_{\alpha}$, and such that for each $\alpha$ there is a function $f_{\alpha} \in H^{\infty}\left(V_{\alpha} \cap U^{n}\right)$ with the same zeros as $f$ in $V_{\alpha} \cap U^{n}$. Let $W$ be an open set satisfying $Z(f) \subset W \subset \bar{W} \subset \cup V_{\alpha}$; then the functions $f_{\alpha}$ together with the constant function $e(z)=1, \forall z \in U^{n}-\bar{W}$, constitute data for Theorem (3.5). This can be seen since it is possible to find a refinement of the covering $\left\{V_{\alpha}, \mathbf{C}^{n}-\bar{W}\right\}_{\alpha \in A}$ of $\bar{U}^{n}$ consisting of products of simply connected domains, and on the overlap of any two of these, the quotient of the corresponding bounded holomorphic functions will be an invertible element of the corresponding Nevanlinna class. The solution of the multiplicative Cousin problem then provides us with a function $F \in N\left(U^{n}\right)$ with the same zeros as $f$.

Our goal now is to find geometric conditions on $Z(f)$ so as to assure that it is locally given by bounded holomorphic functions.

(4.4) THEOREM. Let $n \geqslant 2$ and let $f \in H\left(U^{n}\right)$. If there exist a constant $0<r<1$ and a continuous function $\eta:[r, 1) \rightarrow[r, 1)$, such that

$$
\left|\lambda_{n}\right| \leqslant \eta\left(\frac{\left|\lambda_{1}\right|+\cdots+\left|\lambda_{n-1}\right|}{n-1}\right)
$$

for all points $\left(\lambda_{1}, \cdots, \lambda_{n}\right) \in Z(f) \cap Q^{n}(r, 1)$, there is a function $F \in N\left(U^{n}\right)$ with the same zeros as $f$.

Proof. Fix $r<r^{\prime}<1$ and let

$$
c=\sup \left\{\eta(x): r \leqslant x \leqslant 1-\left(1-r^{\prime}\right) /(n-1)\right\} .
$$

If $\left(\lambda_{1}, \cdots, \lambda_{n}\right) \in Z(f) \cap\left[Q^{i-1}(r, 1) \times Q\left(r, r^{\prime}\right) \times Q^{n-i}(r, 1)\right]$ for any $1 \leqslant$ $i \leqslant n-1$, we have

$$
\left|\lambda_{n}\right| \leqslant \eta\left(\frac{\left|\lambda_{1}\right|+\cdots+\left|\lambda_{n-1}\right|}{n-1}\right) \leqslant c<1 .
$$

Therefore $Z(f) \cap\left[Q^{i-1}(r, 1) \times Q\left(r, r^{\prime}\right) \times Q^{n-i-1}(r, 1) \times Q(c, 1)\right]=\varnothing$. This says that the zero set of $f$ in $U^{i-1} \times U\left(r^{\prime}\right) \times U^{n-i}$ is a Rudin variety $\left(Z(f)\right.$ is bounded away from the essential boundary $T^{i-1} \times T\left(r^{\prime}\right) \times T^{n-i}$ of $\left.U^{i-1} \times U\left(r^{\prime}\right) \times U^{n-i}\right)$. Thus, for each $1 \leqslant i \leqslant n-1$, there exists a function $F_{i} \in H^{\infty}\left(U^{i-1} \times U\left(r^{\prime}\right) \times U^{n-i}\right)$ with the same zeros as $f$ in $U^{i-1} \times$ $U\left(r^{\prime}\right) \times U^{n-i}$. 
The polydomains $\left\{U^{i-1} \times U\left(r^{\prime}\right) \times U^{n-i}\right\}_{1 \leqslant i \leqslant n-1}$ do not cover all of $U^{n}$. However if we add to this collection the polydomain $Q^{n-i}(r, 1) \times U$, we do have an open covering of $U^{n}$. It is clear that we can enlarge these sets so that they determine an open covering of $\bar{U}^{n}$ and such that the intersection of the enlargement of each open set with $U^{n}$ is the given open set.

Our next step is to construct a function $\phi \in H^{\infty}\left(Q^{n-1}(r, 1) \times U\right)$ which recaptures the zeros of $f$ in $Q^{n-1}(r, 1) \times U$.

If we fix $z^{\prime} \in Q^{n-1}(r, 1)$, then the function $f\left(z^{\prime}, \cdot\right)$ has finitely many zeros in $U$, denote these by $\alpha_{1}\left(z^{\prime}\right), \cdots, \alpha_{k\left(z^{\prime}\right)}\left(z^{\prime}\right)$, counted according to multiplicities, and define

$$
\phi(z)=\prod_{i=1}^{k\left(z^{\prime}\right)}\left(z_{n}-\alpha_{i}\left(z^{\prime}\right)\right) \quad\left(z=\left(z^{\prime}, z_{n}\right) \in Q(r, 1) \times U\right) .
$$

Clearly $\phi$ is bounded in its domain. Our goal will be to show that it is holomorphic and with the same zeros as $f$. The proof will be similar to that of the Weierstrass preparation theorem.

Let $\rho$ be arbitrarily chosen so that $r<\rho<1$, and let $d=\sup \{\eta(x)$ : $r \leqslant x \leqslant \rho\}$. Define for each $p=0,1,2, \cdots$ and $z^{\prime} \in Q^{n-1}(r, \rho)$,

$$
S_{p}\left(z^{\prime}\right)=\frac{1}{2 \pi i} \int_{|\xi|=d} \frac{\operatorname{Dn} f\left(z^{\prime}, \xi\right)}{f\left(z^{\prime}, \xi\right)} \xi^{p} d \xi,
$$

where $D n$ denotes differentiation with respect to $z_{n}$. Note that $S_{0}\left(z^{\prime}\right)$ is the number of zeros of $f\left(z^{\prime}, \cdot\right)$ in $U$ (there are only finitely many since they all must lie outside of $\overline{U(d))}$. Also observe that the functions $S_{p}$ are holomorphic in $Q^{n-1}(r, \rho)$. Since $S_{0}$ is an integer valued continuous function, it must be a constant; i.e., $S_{0}\left(z^{\prime}\right)=k$ for all $z^{\prime} \in Q^{n-1}(r, \rho)$. Then in (4.4.1) $k\left(z^{\prime}\right)$ is independent of $z^{\prime}$.

For fixed $z^{\prime}, \phi(z)$ is a polynomial in $z_{n}$, which can be written in the form

$$
\phi(z)=z_{n}^{k}+b_{1}\left(z^{\prime}\right) z_{n}^{k-1}+\cdots+b_{k}\left(z^{\prime}\right) .
$$

We must show that each $b_{i}$ is holomorphic in $Q^{n-1}(r, \rho)$. But $S_{p}\left(z^{\prime}\right)=$ $\alpha_{1}\left(z^{\prime}\right)^{p}+\cdots+\alpha_{k}\left(z^{\prime}\right)^{p}$ is holomorphic in $Q^{n-1}(r, \rho)$, and for each $1 \leqslant$ $i \leqslant k-i b_{i}=S_{i}+S_{i-1} b_{1}+\cdots+S_{1} b_{i-1}$ (Newton's identities). Thus $b_{1}$, $\cdots, b_{k}$ are holomorphic in $Q^{n-1}(r, \rho)$.

Since for every $\rho<1, \phi(z)$ is holomorphic in $Q(r, \rho)^{n-1} \times U$, it follows that it must be holomorphic in $Q(r, 1)^{n-1} \times U$. By its definition it is clear that $\phi$ has the same zeros as $f$ in $Q^{n-1}(r, 1) \times U$; i.e. $f \phi^{-1}$ is a zero free holomorphic function.

Since $f \phi^{-1}$ and $f F_{i}^{-1}, 1 \leqslant i \leqslant n-1$, are zero free holomorphic func- 
tions, the same will be the case with $F_{i} F_{j}^{-1}$ and $F_{i} \phi^{-1}$. Thus, by (4.4.3), for all $1 \leqslant i, j \leqslant n-1, F_{i} F_{j}^{-1}$ and $F_{i} \phi^{-1}$ will be invertible elements of the corresponding Nevanlinna classes. Theorem (3.5) then shows that there exists a function $F \in N\left(U^{n}\right)$ with the same zeros as $f$ in $U^{n}$.

REMARKS. If $\eta(x)$ is bounded away from 1 , then $Z(f)$ is a Rudin variety, and therefore there exists $F \in H^{\infty}\left(U^{n}\right)$ with the same zeros as $f$.

The conclusion of (4.4) would be the same if we replace the condition

$$
\left|\lambda_{n}\right| \leqslant n\left(\frac{\left|\lambda_{1}\right|+\cdots+\left|\lambda_{n-1}\right|}{n-1}\right)
$$

by

$$
\left|\lambda_{k}\right| \leqslant \eta\left(\frac{\left|\lambda_{1}\right|+\cdots+\left|\lambda_{k-1}\right|+\left|\lambda_{k+1}\right|+\cdots+\left|\lambda_{n}\right|}{n-1}\right)
$$

for some $1 \leqslant k \leqslant n$, and all points $\left(\lambda_{1}, \cdots, \lambda_{n}\right) \in Z(f) \cap Q^{n}(r, 1)$.

The condition of Theorem (4.4) is not a necessary one, for if $B(z)$ is an infinite Blaschke product and $f\left(z_{1}, z_{2}\right)=B\left(z_{1}\right)$ then the zero set of $f$ does not satisfy this condition although $f$ is in $H^{\infty}\left(U^{2}\right)$.

Finally, with regard to Theorem (4.4), an open question is whether the function $F$ can always be chosen in $H^{\infty}\left(U^{n}\right)$.

\section{REFERENCES}

1. L. V. Ahlfors, Complex analysis: An introduction to the theory of analytic functions of one complex variable, 2nd ed., McGraw-Hill, New York, 1966. MR 32 \# 5844.

2. P. S. Chee, On the generalized Blaschke condition, Trans. Amer. Math. Soc. 152 (1970), 227-231. MR 42 \#3303.

3. - The Blaschke condition for bounded holomorphic functions. Trans. Amer. Math. Soc. 148 (1970), 249-263. MR 41 \#7147.

4. W. Rudin, Function theory in polydiscs, Benjamin, New York, 1969. MR 41 \#501.

5. - Zero-sets in polydiscs, Bull. Amer. Math. Soc. 73 (1967), 580-583. MR 35 \#1819.

6. E. L. Stout, The second Cousin problem with bounded data, Pacific J. Math. 26 (1968), 379-387. MR 38 \#3467.

7. S. E. Zarantonello, Sobre los ceros de funciones analiticas acotadas de varias variables complejas, An. Inst. Mat. Univ. Nac. Autónoma México (to appear).

DEPARTMENT OF MATHEMATICS, UNIVERSITY OF FLORIDA, GAINESVILLE, FLORIDA 32601 